\title{
Método de elementos finitos bi e tridimensional para investigação do modo de falha em testes de cisalhamento e microcisalhamento em restaurações dentárias
}

\author{
Two and three-dimensional finite element method \\ to investigate failure mode from shear and \\ micro-shear tests on dental restorations
}

Eric Costa Carvalho ${ }^{1}$, Jayme Pereira de Gouvêa ${ }^{1}$, Ágatha Borges Teixeira ${ }^{1}$, Tereza Cristina Favieri de Melo-Silva ${ }^{2}$, Cláudio Luís de Melo-Silva ${ }^{2}$

\footnotetext{
${ }^{1}$ Universidade Federal Fluminense, Engenharia Metalúrgica e Materiais, Rua 12 - Vila Santa Cecília, CEP: 27255-125, Rio de Janeiro, Volta Redonda, Brasil.

${ }^{2}$ Centro Universitário de Volta Redonda, Odontologia, Avenida Paulo Erlei Alves Abrantes, 1325 - Três Poços, 27240560, Rio de Janeiro, Volta Redonda, Brasil.

e-mail: costa_eric1@ hotmail.com,jpg@metal.eeimvr.uff.br, htaborges@gmail.com, terezafmelo@ig.com.br, claudiolmelo@ig.com.br.
}

\section{RESUMO}

Este estudo analisou, por meio de ensaios de resistência de união ao cisalhamento e microcisalhamento, os tipos de fraturas e verificou a distribuição de tensões na interface por método de elementos finitos. Os modelos foram compostos por dentina, adesivo e resina composta. Como simplificação da metodologia, os materiais foram considerados elástico-lineares e isotrópicos. Dois modelos bi e tridimensionais foram criados para ambos os ensaios; e as forças aplicadas nos testes foram $50 \mathrm{~N}$ para cisalhamento e $15 \mathrm{~N}$ para microcisalhamento. O critério de falha abordado foi o de Mohr-Coulomb que descreve a resposta de

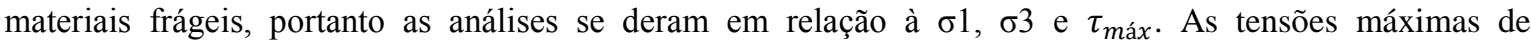
cisalhamento foram comparadas com as tensões nominais dos modelos e observou-se que o modelo de cisalhamento apresenta uma maior variação se comparado ao microcisalhamento, outro ponto importante levantado é que a distribuição de tensões no modelo bidimensional é semelhante ao modelo tridimensional, sendo assim, o modelo pode ser simplificado a uma análise bidimensional nesse aspecto. Embora sejam aplicados esforços cisalhantes, o material falha por tração, fator observado quando analisamos o ensaio de cisalhamento. Ao se analisar graficamente o critério de falha Mohr-Coulomb modificado pôde-se observar possível falha coesiva da dentina para ensaios de cisalhamento e falha adesiva para os ensaios de microcisalhamento, e também os valores de $\tau_{n o m}$ estiveram mais próximos da faixa de $\tau_{\text {máx }}$ para os ensaios de microcisalhamento. Sendo assim, o estudo permite concluir que a metodologia desenvolvida de resistência adesiva para o ensaio de microcisalhamento é validada pela simulação computacional.

Palavras-chave: cisalhamento, microcisalhamento, dentina, resistência adesiva, elementos finitos.

\section{ABSTRACT}

This study analyzed by means of conventional shear and microshear bond strength tests types of fractures and it verified stress distribution in the interface by finite element method. The models were composed of dentin, adhesive and composite resin. As simplification of the methodology the materials were considered elasticlinear and isotropic. Two and three-dimensional models were created for both tests and the forces applied in the tests were $50 \mathrm{~N}$ for shear and $15 \mathrm{~N}$ for microshear. The failure criterion approached was Mohr-Coulomb that describes the response of fragile materials, so the analyses were given in relation to $\sigma 1, \sigma 3 \mathrm{e} \tau_{\max }$. The maximum shear stresses were compared with the nominal stresses of the models and it was observed that the shear model has a greater variation compared to microshear. Another important point raised is that tensions distribution in the two-dimensional model is similar to the three-dimensional model, thus, the model can be simplified to a two-dimensional analysis in this aspect. Although shear stresses are applied, the material 
failed by traction, a factor observed when analyzing the shear test. By graphically analyzing the modified Mohr-Coulomb failure criterion, it was possible to observe a cohesive dentin failure for shear test and adhesive failure for microshear test, and also the $\tau_{\text {nom }}$ values were closer to $\tau_{\max }$ microshear test. Thus, the study allows the conclusion that the methodology developed of adhesive resistance for the microshear test is validated by computational simulation.

Keywords: shear, micro-shear, dentin, bond strength, finite element analysis.

\section{INTRODUÇÃO}

Sistemas adesivos e compósitos resinosos foram os materiais odontológicos que mais sofreram melhorias nas últimas décadas. Apesar deste considerável progresso de adesão destes compósitos aos substratos dentários, procuram-se materiais restauradores que, além de serem biologicamente compatíveis com a estrutura dentária, possuam resistência suficiente para suportar os esforços mastigatórios [1,2]. Problemas técnicos relacionados à utilização dos sistemas adesivos estão associados principalmente às características morfológicas e de composição dos substratos aderentes, sobretudo à complexidade da dentina, pois em esmalte é uma técnica mais segura e duradoura [3, 4].

A resistência de união entre material resinoso e substrato dentário pode ser medida por diversos métodos, como o de cisalhamento e tração. Esses testes se baseiam na aplicação de uma força, que cria tensões na interface de união até que uma falha seja observada. O teste de força de união é definido como o teste mecânico projetado para medir a tensão necessária para interromper a ligação entre dois materiais [5, 6].

O teste de cisalhamento é um dos mais simples e amplamente utilizado. Neste teste, a interface é rompida por uma força aplicada paralelamente à interface adesiva, estando o corpo-de-prova de maneira perpendicular à ação da força. Para realização deste ensaio são utilizados espécimes em forma de cilindro e com diâmetro variando de 2 a $4 \mathrm{~mm}$ sendo estes aderidos a um substrato plano por meio de um adesivo [7].

A simplicidade do teste de resistência de união (ou adesiva), apesar de ser fortemente criticada, tornou-se um procedimento rotineiro para a determinação da eficácia da ligação sendo utilizado para medidas de comparação clínica e até mesmo de marketing empresarial para venda de produtos com maior performance [8]. O valor de resistência adesiva obtido em testes experimentais é uma tensão calculada dividindo-se a carga suportada na fratura pela área da seção transversal da interface aderida. Este valor, denominado tensão nominal ou média, não é capaz de exprimir eventuais picos de tensão suportados pela interface no momento da ruptura. Portanto, nota-se a necessidade de uma análise mais detalhada da distribuição de tensões ao longo dessa interface adesiva, sendo esta realizada por Elementos Finitos [9].

A principal dificuldade associada à interpretação dos dados do teste de ligação é a falta de informações sobre a distribuição de tensões e os tipos de tensão gerados em cada espécime de teste. O método de elementos finitos (MEF) tem sido utilizada em várias ocasiões para calcular os estados de tensões em muitas das geometrias associadas à resistência de ligação relatadas na literatura [10]. Esta análise numérica por simulação computacional pode-se observar, além da distribuição não-uniforme de tensões, uma complexa variação das mesmas, ou seja, as tensões não sendo exclusivamente trativas ou compressivas, por exemplo. Em outras palavras, em alguns casos, embora o carregamento seja influenciado por um tipo de tensão o material pode fraturar por outro [11].

A análise por Elementos Finitos também é uma importante ferramenta para explicar a ocorrência de fraturas e discutir a validade dos valores de resistência adesiva nominal obtidos nos ensaios mecânicos, uma vez que fornecem informação sobre a natureza e o estado de tensões nas várias partes da amostra simulada, podendo indicar os sítios mais prováveis de início de uma fratura, ou seja, locais de maior concentração de tensão $[12,13]$.

Sendo assim, o presente estudo tem por objetivo analisar e comparar os critérios de falha dos testes de cisalhamento e microcisalhamento bi e tridimensionalmente por meio de métodos de elementos finitos.

\section{MATERIAIS E MÉTODOS}

O software utilizado para realizar a análise de Elementos Finitos foi o Ansys ${ }^{\circledR}$ Mechanical APDL para a etapa de pré, processamento e pós-processamento. O acesso foi disponibilizado pela Universidade Federal Fluminense (UFF). 


\subsection{Modelo bidimensional}

Como simplificação da metodologia, os materiais foram considerados elástico-lineares e isotrópicos. Para este estado plano de tensões o tipo de elemento adotado foi o Plane $182 \mathrm{com}$ malhas de 0,04 $\mathrm{mm}$ para todos os materiais. O elemento é definido por quatro nós com dois graus de liberdade em cada nó. As malhas foram refinadas até que se comprovasse que a solução computacional não variava com o aumento do refino, por meio do teste de convergência de malha. Foi realizado um merging durante a modelagem da camada adesiva entre dentina e resina composta e o modelo geométrico final foi composto por 12.198 elementos para cisalhamento e 10.384 para microcisalhamento. A representação dos ensaios mecânicos está evidenciada na Figura 1. Para condição de contorno considerou-se a dentina engastada na parte inferior, superior e posterior conforme Figura 2 (a). A diferença entre os testes se dá principalmente na área aderida, uma vez que valores inferiores a $1 \mathrm{~mm}^{2}$ já são considerados ensaios de microcisalhamento.

As dimensões da dentina foram estabelecidas como 10 x $5 \mathrm{~mm}$, o sistema adesivo com uma espessura de $0,05 \mathrm{~mm}$ e o pino resinoso para cisalhamento $2 \times 2 \mathrm{~mm}$ e para microcisalhamento $2 \times 1 \mathrm{~mm}$. Ao se fixar o pino resinoso à dentina, o adesivo que é responsável por este selamento se espalha e nas bordas de contato gera uma angulação devido ao molhamento. Para análise de elementos finitos este fator pode ser preponderante na distribuição de tensões locais da amostra, e assim, variando seu modo de falha. A angulação considerada foi de $45^{\circ}$. Uma fratura foi levada ao Microscópio Confocal Interferométrico (Leica DCM 3D) para realização de uma análise topográfica. Esta análise consiste em dispor uma linha que define a rugosidade sobre a imagem. A linha deve ser traçada de maneira radial de modo a identificar os relevos da amostra. Por fim, um gráfico foi gerado pelo programa e neste pôde-se aferir a angulação do filete resino, sendo esta angulação utilizada para elaborar os modelos bi e tridimensionais.
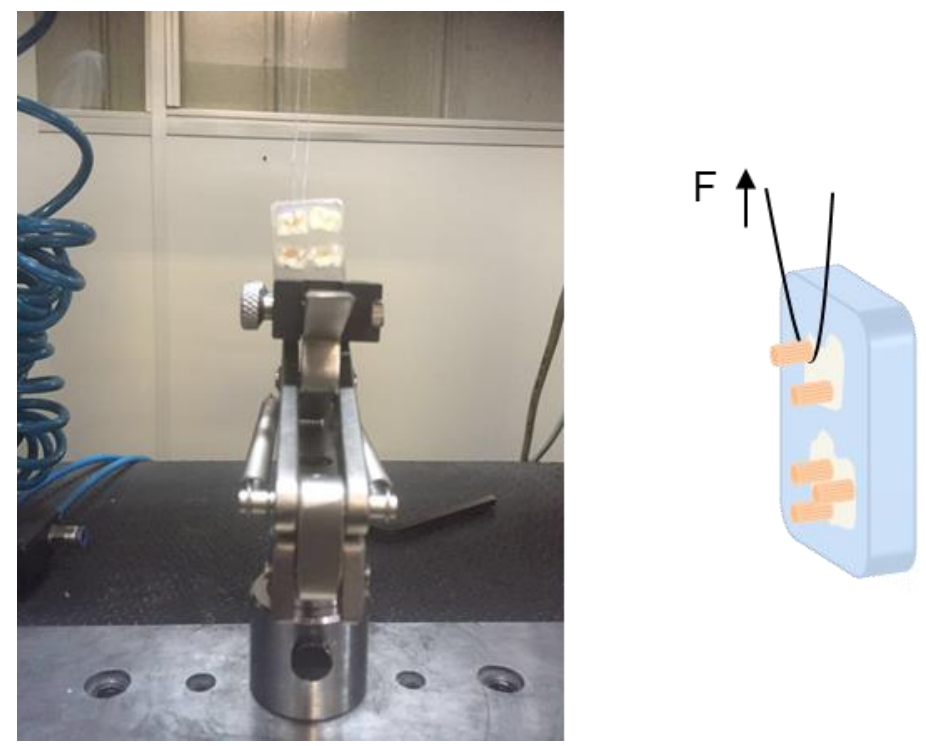

Figura 1: Imagens fotográfica e esquemática dos ensaios mecânicos realizados no equipamento EMIC DL2000 para suporte dos modelos de elementos finitos.

Para o desenvolvimento dos modelos foram utilizados valores de módulo de elasticidade encontrados pela equipe de Biomateriais UFF por ensaios de ultramicrodureza (Resina Composta Filtek Z350 XT ® e Adesivo Single Bond Universal ${ }^{\circledR}$ - 3M ESPE) e os coeficientes de Poisson de acordo com PLÁCIDO et al. [13] estando descritos na Tabela 1. Os valores também foram aplicados no modelo tridimensional. 
Tabela 1: Propriedades mecânicas e dimensões dos materiais utilizados.

\begin{tabular}{|c|c|c|c|c|}
\hline \multirow{2}{*}{ Materiais } & \multicolumn{2}{|c|}{ Propriedades } & \multicolumn{2}{|c|}{$\begin{array}{c}\text { Dimensões (mm) } \\
\text { comprimento x largura }\end{array}$} \\
\hline & $\begin{array}{c}\text { Módulo de } \\
\text { Elasticidade (GPa) }\end{array}$ & $\begin{array}{l}\text { Coeficiente de } \\
\text { Poisson }\end{array}$ & G1 & G2 \\
\hline Dentina & 20 & 0,23 & $10 \times 5$ & $10 \times 5$ \\
\hline Resina Composta & 11 & 0,25 & $2 \times 2$ & $2 \times 1$ \\
\hline Adesivo & 5 & 0,35 & $0,05 \times 2$ & $0,05 \times 1$ \\
\hline
\end{tabular}

Para aplicação da carga no modelo foi considerada a carga média dos ensaios laboratoriais, $50 \mathrm{~N}$ para cisalhamento e $15 \mathrm{~N}$ para microcisalhamento. A carga uniaxial foi aplicada a $0,2 \mathrm{~mm}$ (simulando a espessura do fio ortodôntico) no sentido positivo do eixo y decomposta em 5 vetores próximos aos nós da interface sendo que o segmento $\mathrm{AB}$ representa a interfaces analisada na distribuição de tensões conforme demonstrado na Figura 2 (b).
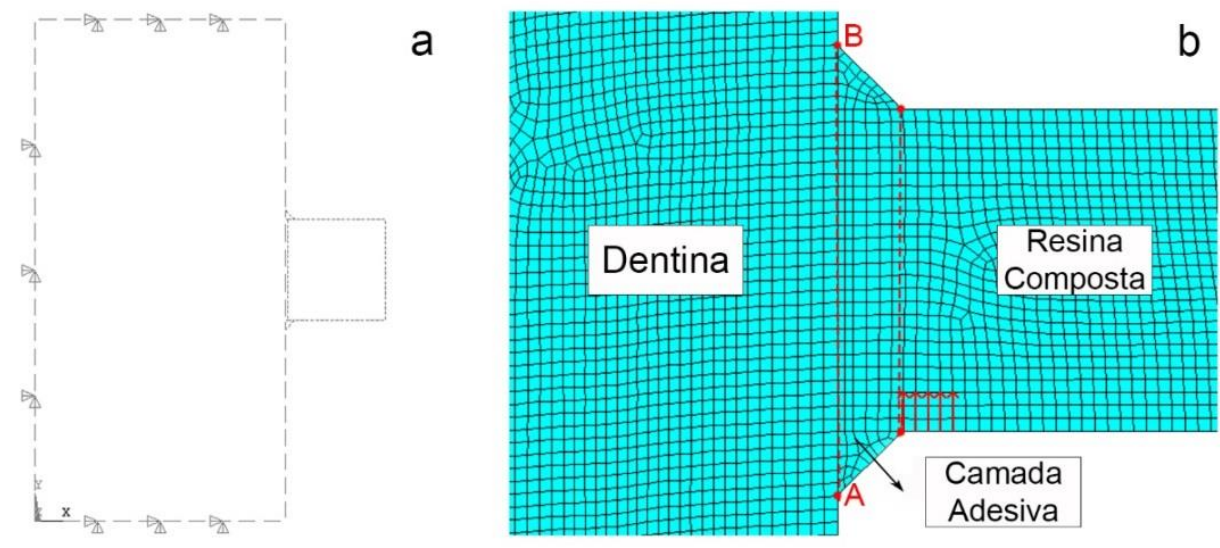

Figura 2: a) Imagem bidimensional representativa do ensaio de cisalhamento. As linhas pontilhadas demarcam os limites dos materiais utilizados e setas representam os pontos de engaste da dentina; e b) Distribuição das cargas (setas) ao longo da superfície resina composta representando o sentido da aplicação da força e a linha de análise da interface AB. Imagem Ansys.

\subsection{Modelo tridimensional}

O elemento utilizado foi o Solid187, tendo um comportamento de deslocamento quadrático, com 162.527 elementos para cisalhamento e 197.382 para microcisalhamento. O elemento é definido por 10 nós com três graus de liberdade em cada nó. Para condição de contorno a dentina foi considerada engastada na parte inferior, superior e posterior. As dimensões da dentina foram estabelecidas como 5 x 10 x $5 \mathrm{~mm}$, o sistema adesivo com uma espessura de $0,05 \mathrm{~mm}$ e o pino resinoso pino resinoso com $1 \mathrm{~mm}$ de diâmetro para microcisalhamento e $2 \mathrm{~mm}$ para cisalhamento e ambos com $2 \mathrm{~mm}$ de comprimento. As cargas para o desenho tridimensional foram distribuídas de acordo com a decomposição de forças em 17 nós distintos ao longo da interface de modo a representar o contato com o fio conforme demonstrado na Figura 3, seguindo a mesma ideia do modelo bidimensional de $50 \mathrm{~N}$ para cisalhamento e $15 \mathrm{~N}$ para microcisalhamento. Para simular o fio ortodôntico as cargas foram distribuídas conforme a decomposição das forças normais (FN) em componentes horizontais $(\mathrm{Fx})$ e verticais $(\mathrm{Fy})$ ao longo de $180^{\circ}$. O modelo geométrico final foi composto por 39.743 elementos para cisalhamento e 39.494 para microcisalhamento. Para condição de contorno considerou-se a dentina engastada na parte inferior, superior e posterior como no modelo bidimensional. 

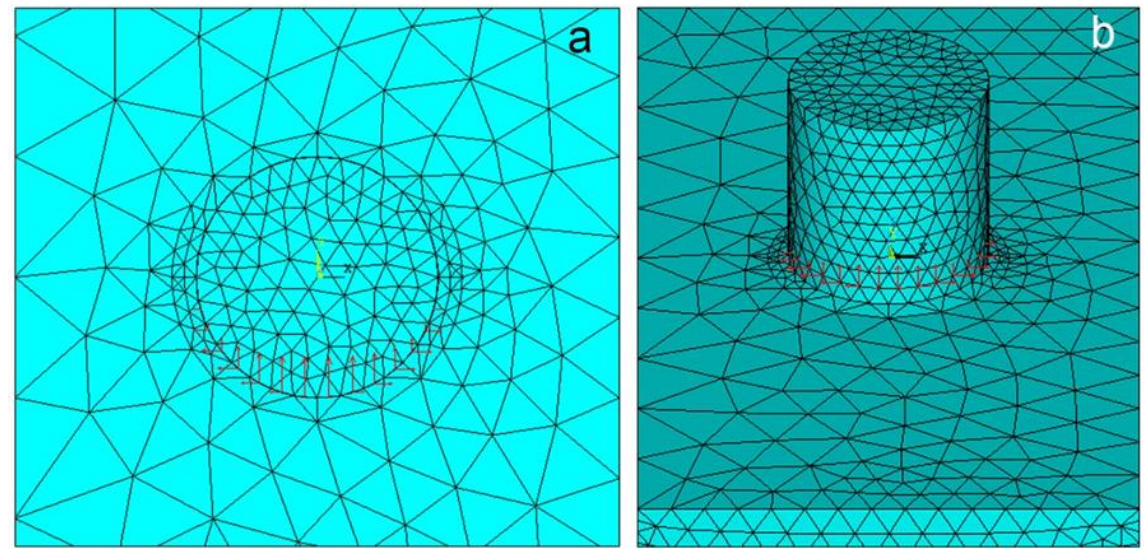

Figura 3: Distribuição das cargas (setas) ao longo da interface representando os esforços cisalhantes no desenho tridimensional para microcisalhamento. Imagem Ansys.

\section{RESULTADOS E DISCUSSÃO}

Ao se realizar os ensaios mecânicos, as tensões de ruptura obtidas nos mesmos representam uma tensão média, não levando em consideração a distribuição de tensões ao logo do material restaurado. Van Noort, CARDEW e HOWARD [14] analisaram que os ensaios ofereciam apenas um valor nominal para a força de ligação, pois é calculado a partir do valor da carga aplicada na ruptura dividida pela área superfície ligada. Essas tensões locais podem ser muito superiores aos valores brutos ou nominais, pois a distribuição de tensões na interface está longe de ser uniforme. Sendo assim, verificou-se a necessidade de uma análise por MEF que nos permite determinar valores locais reais de tensão na linha de união de qualquer arranjo de teste estabelecido. Embora este seja delimitado pelas características do modelo gerado, fornece uma análise útil do que se passa na interface ligada de uma geometria complexa na presença de uma carga aplicada.

AUSIELLO, APICELLA e DAVIDSON [15] realizaram estudos de elementos finitos para verificar o efeito das propriedades da camada adesiva na distribuição de tensões em um dente restaurado, abordando 3 tipos de carga oclusal distintas. O critério de falha abordado foi o de von Mises, sendo este aplicável a materiais dúcteis. Sabe-se que o dente, bem como seus materiais restauradores quando submetidos a um carregamento se comportam de maneira frágil. Sendo assim, a análise deste projeto é baseada nas tensões principais e não de acordo com os critérios de von Mises.

No modelo analisado no presente projeto, está demonstrada na Figura 3 (a) a distribuição de tensões locais para os ensaios de cisalhamento no modelo bidimensional referentes à primeira tensão principal ou $\sigma 1$ com variação máxima de $129 \mathrm{MPa}$; e na Figura 4 (b) referente ao modelo bidiminesional de microcisalhamento com variação até $53 \mathrm{MPa}$. A Figura 4 (c) e (d) representam a variação dos valores no modelo tridimensional para cisalhamento (c) até $47 \mathrm{MPa}$ e microcisalhamento (d) com variação de $\sigma 1$ aproximada do modelo bidimensional de até $69 \mathrm{MPa}$. A diferença na distribuição se dá principalmente à geometria dos modelos. O estado plano de tensões considera a terceira dimensão como unitária, transformando assim o cilindro em uma barra e afetando principalmente o cálculo da tensão nominal. Porém, algumas características se assemelham em ambos os modelos, como o valor máximo de tensão no ponto de aplicação da força, a variação de tensões partindo da interface para a dentina e presença de forças compressivas no lado oposto à aplicação da carga [16].

De acordo com VAN NOORT et al. [17] existe uma maior concentração de tensão no ângulo entre compósito e adesivo e isso pode ser um indicativo de que a fratura começa nesse local. Afirmaram ainda que os valores obtidos são mais realistas quando se analisa a interface dentro dos limites próximos ao cilindro. Já em estudos de XAVIER et al. [18], não houve delimitação em nenhum ensaio, principalmente pela dificuldade em se estabelecer uma delimitação perfeita para as amostras de tamanho micro. Relataram também que ao delimitar a extensão do adesivo sob o cilindro poderia funcionar como um concentrador de tensões na interface, sendo fonte de defeito em uma área crítica do modelo. 

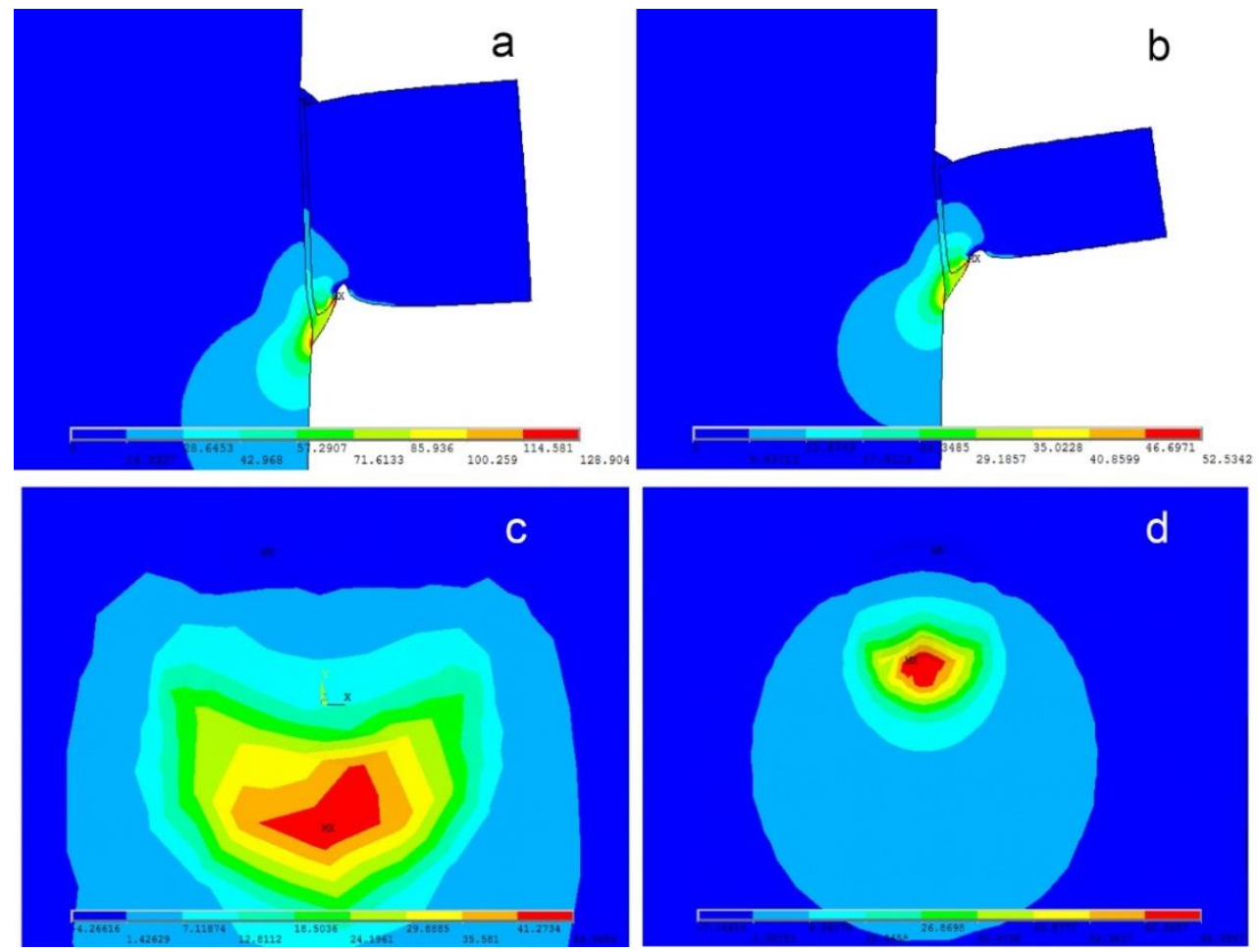

Figura 4: Tensões principais $\sigma 1$ para ensaios de cisalhamento (a) e microcisalhamento (b) em estado plano de tensões; cisalhamento (c) e microcisalhamento (d) no modelo tridimensional. Simulação em MEF.

A tensão máxima de $\sigma 1$ apresenta ponto máximo no local de aplicação da força e mínimo no lado oposto à aplicação devido à natureza do ensaio proposto. Para analisar o que ocorre na interface foram traçadas duas linhas $\mathrm{AB}$ ao longo da mesma de modo a verificar o perfil de tensões e gerar um gráfico das tensões cisalhantes. As curvas levantadas estão evidenciadas na Figura 4 sendo que a distância zero representa o ponto de aplicação da carga. Estão representadas as curvas das tensões cisalhantes obtidas no presente estudo ao longo das interfaces, em que o $\tau_{\text {máx }}$ representa as tensões de cisalhamento máximas sendo obtido pela equação $\quad \tau_{\text {máx }}=\frac{(\sigma 1-\sigma 3)}{2}$, em que $\sigma 1$ representam as máximas tensões principais e $\sigma 3$ as mínimas. De acordo com a Figura 4 (a), para o ensaio de cisalhamento, os pontos máximos de tensão cisalhante é em torno de $50 \mathrm{MPa}$ e na Fig. 4 (b), para o ensaio de microcisalhamento, varia entre $20 \mathrm{MPa}$ a 28 MPa.

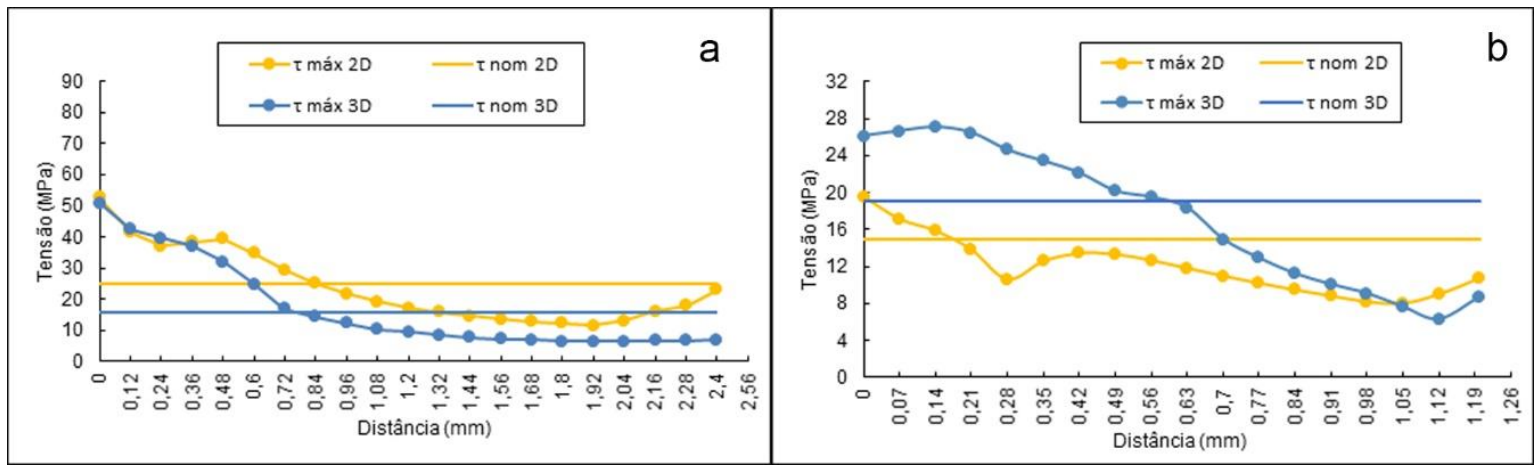

Figura 5: Gráfico da linha de interface $\mathrm{AB}$ pino/dentina evidenciando as tensões cisalhantes para ensaios de (a) cisalhamento e (b) microcisalhamento. A distância 0 representa o ponto de aplicação da carga. 
Para dar continuidade no aprofundamento da discussão, foi realizado um compilado dos principais resultados sobre o tema estudado no que diz respeito à variação de tensões ao longo da interface $\mathrm{AB}$. Estão evidenciados na Tabela 2 os valores referentes a tensões principais, máximas cisalhantes, nominais e variações de acordo com os modelos bi e tridimensionais.

Tabela 2: Compilado dos resultados de tensões dos principais autores referentes ao tema estudado. Tensões máximas ocorridas na interface $\mathrm{AB}$.

\begin{tabular}{|c|c|c|c|c|c|c|}
\hline & \multirow{2}{*}{$\begin{array}{c}\text { Tensão } \\
\text { (MPa) }\end{array}$} & \multicolumn{2}{|c|}{ Cisalhamento } & \multicolumn{2}{|c|}{ Microcisalhamento } & \multirow{2}{*}{$\begin{array}{c}\text { Módulo de } \\
\text { Elasticidade do } \\
\text { Compósito (GPa) }\end{array}$} \\
\hline & & 2D & $3 \mathrm{D}$ & 2D & $3 \mathrm{D}$ & \\
\hline \multirow{4}{*}{ 음 } & $\mathrm{S} 1$ & 105 & 104 & 39 & 48 & \multirow{4}{*}{11} \\
\hline & S3 & 0 & 3 & 0 & -6 & \\
\hline & $\tau$ máx & 53 & 51 & 20 & 27 & \\
\hline & $\tau$ nom & 25 & 16 & 15 & 19 & \\
\hline \multirow{3}{*}{ 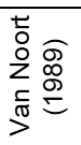 } & S1 & 150 & & & & \multirow{3}{*}{25} \\
\hline & S3 & & & & & \\
\hline & $\begin{array}{l}\tau \text { máx } \\
\tau \text { nom }\end{array}$ & 10 & & & & \\
\hline \multirow{8}{*}{ 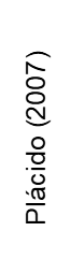 } & S1 & 35 & & 23 & & \multirow{4}{*}{20} \\
\hline & S3 & & & & & \\
\hline & $\tau$ máx & 12 & & 10 & & \\
\hline & $\tau \mathrm{nom}$ & 5 & & 5 & & \\
\hline & S1 & 70 & & 45 & & \multirow{4}{*}{5} \\
\hline & S3 & & & & & \\
\hline & $\tau$ máx & 25 & & 15 & & \\
\hline & $\tau$ nom & 5 & & 5 & & \\
\hline \multirow{8}{*}{ 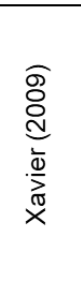 } & S1 & & 56 & & 24 & \multirow{4}{*}{20} \\
\hline & S3 & & & & & \\
\hline & $\tau$ máx & & 49 & & 7 & \\
\hline & $\tau$ nom & & 4 & & 4 & \\
\hline & S1 & & 82 & & 17 & \multirow{4}{*}{5} \\
\hline & S3 & & & & & \\
\hline & $\tau$ máx & & 60 & & 10 & \\
\hline & $\tau$ nom & & 4 & & 4 & \\
\hline \multirow{3}{*}{ 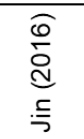 } & S1 & & 145 & & & \multirow{3}{*}{15} \\
\hline & S3 & & & & & \\
\hline & $\begin{array}{l}\tau \text { máx } \\
\tau \text { nom }\end{array}$ & & 45 & & & \\
\hline
\end{tabular}

Os dados que variam nos estudos são, principalmente, o tipo de compósito estudado e o ponto de aplicação da carga, sendo que na Tabela 2 foram consolidados os valores de pontos de aplicação semelhantes aos do presente estudo, variando somente os tipos de compósito. Os autores extraíram os valores de $\tau_{\text {máx }}$ diretamente do software de simulação, não passando pela etapa de análise de S3, e adotando assim, $\mathrm{S} 1=$ $\mathrm{S}_{\text {máx }}$. Essa diferença se dá porque os autores realizaram a análise por von Mises modificado. De acordo com MISRA et al. [20] esse parâmetro de análise poderia ser mais coerente para a predição das fraturas por meio do MEF. Justificaram também, que por não ser claro o mecanismo de falha da interface, além da análise pelas tensões principais, a análise por von Mises seria válida para comparação.

No presente estudo, para os ensaios de cisalhamento, a tensão nominal $\tau_{\text {nom }}$ representa a tensão cisalhante média que é constante ao longo da interface sendo calculada por $\tau_{\text {nom }}=\frac{F}{A}$ onde F representa a força do ensaio e A à área transversal da aplicação da força, o valor encontrado foi de $25 \mathrm{MPa}$ para o modelo bidimensional e $16 \mathrm{MPa}$ para o modelo tridimensional. Isso justifica o ponto de que as tensões locais são muitos maiores do que as tensões nominais, ocasionando uma não-uniformidade na distribuição de tensões ao longo da interface. Para microcisalhamento o valor de tensão nominal encontrado foi de $15 \mathrm{MPa}$ para o modelo bidimensional e $19 \mathrm{MPa}$ para o modelo tridimensional. Isso significa que a tensão nominal para os ensaios de microcisalhamento está de acordo com os resultados do modelo de simulação, diferentemente do ocorrido para os ensaios de cisalhamento em que o $\tau_{\text {máx }}$ é relativamente maior que o $\tau_{\text {nom }}$, o que poderia levar a uma invalidação do modelo. O teste de cisalhamento convencional tem sido criticado há muito tempo por não ser apropriado ou confiável para verificar a chamada força de adesão real [21]. Outro ponto importante levantado é que a distribuição de tensões no modelo bidimensional é semelhante ao modelo tridimensional, sendo assim, o modelo pode ser simplificado a uma análise bidimensional nesse aspecto. Para tanto, foi realizado um teste estatístico de comparação múltipla de Tukey com nível de significância de 5\%, 
de modo a avaliar a diferença entre os grupos e não houve diferença significativa entre os valores das tensões máximas cisalhantes e nominais para os modelos bi e tridimensional.

Alguns autores avaliam o critério de falha pela razão entre tensão principal máxima e tensão cisalhante máxima, caso essa razão seja maior que 1 o modo de falha será tração, caso seja menor que 1 o modo de falha será cisalhamento [13]. Ao realizar os ensaios mecânicos, o rompimento ocorrido na estrutura restaurada é instantâneo e, portanto, este estudo propôs a análise por um gráfico que representa o critério de falha denominado Mohr-Coulomb modificado conforme Figura 6 (a) e (b). A teoria Mohr-Coulomb é um modelo matemático que descreve a resposta de materiais quebradiços ou frágeis, como concreto e tijolo, tanto à tensão de cisalhamento quanto à tensão normal. A maioria dos materiais clássicos de Engenharia, de alguma forma, segue essa regra em pelo menos uma parte do envelope de sua falha por cisalhamento. Geralmente, a teoria se aplica a materiais para os quais a resistência à compressão excede em muito a resistência à tração [22]. Cada um dos campos coloridos do gráfico representa determinado modo de falha e os limites destes estão estabelecidos pela tensão de tração (S1) e compressão (S3) da dentina, sendo estas 100 MPa e - $300 \mathrm{MPa}$, respectivamente, extraídas de estudos de Sakaguchi e Powers [23]. As coordenadas de S1 e S3 foram retiradas do ponto crítico da Figura 5, sendo que coordenada marcada com um "x" em vermelho representa o ponto da interface onde se inicia a fratura de acordo com as tensões máximas cisalhantes. $\mathrm{O}$ ensaio de cisalhamento está representado na Figura 6 (a) e evidencia que houve falha por tração na dentina, uma vez que as coordenadas estão fora da zona segura estando de acordo com estudos de PLÁCIDO et al. [13], JIN et al. [19] e XAVIER e BALLESTER [21]. Já o gráfico do critério de falhas para os ensaios de microcisalhamento está evidenciado na Figura 6 (b). Nota-se que os dois modelos se encontram na zona segura do gráfico, ou seja, a dentina não fratura. Estas coordenadas, provavelmente, denotam os valores de resistência adesiva e ajudam a confirmar a ideia de que esta resistência adesiva foi validada pelo método de elementos finitos.

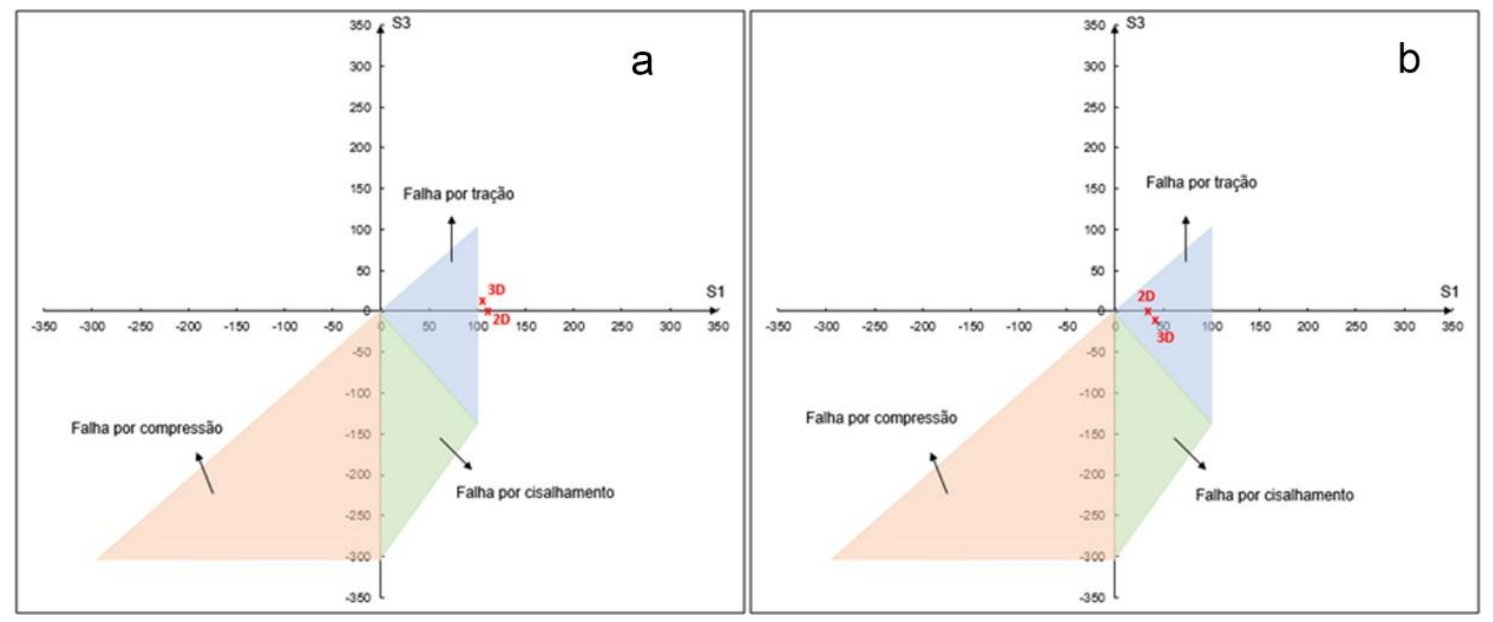

Figura 6: Critério de falha Mohr - Coulomb modificado para (a) cisalhamento e (b) microcisalhamento. Análise bi e tridimensional para a interface AB. Valores de tensão representador em MPa.

Com uma linha semelhante de análise da interface, XAVIER e BALLESTER [21] também encontraram o modo de falha por tração. Ressaltaram ainda sobre a tendência de fraturas coesivas (quando ocorre fratura da dentina ou da resina), que afirmam ocorrer na dentina devido à concentração de tensões no substrato e especialmente à tensão de tração. Os resultados mostram que a tensão principal máxima é encontrada no ângulo entre o adesivo e a dentina, o que sugere uma soma de dois fatores de concentração de tensão: uma angulação acentuada e a proximidade da região de aplicação de carga.

As análises sobre a distribuição de tensões em testes de cisalhamento convencionais não podem ser aplicadas aos de microcisalhamento. Uma das razões pode ser a falta de proporcionalidade entre os aspectos geométricos de ambos os testes, enquanto os diâmetros dos pinos resinosos ou da dentina podem variar, a espessura da camada adesiva sempre permanecerá constante e, portanto, a necessidade da análise de elementos finitos [13].

Os resultados dos ensaios de resistência de união ao cisalhamento dependem principalmente da área testada, e é mais provável achar um defeito que inicia a fratura em uma área maior do que em uma menor. Portanto, a resistência adesiva de um material diminui à medida que o tamanho da área testada aumenta, pois, 
qualquer defeito que exista na interface poderá se propagar facilmente [24]. Outro ponto importante é o ponto de aplicação da carga na relação de concentração de tensões na interface. A explicação desse fenômeno se dá pelo princípio de Saint-Venant, pois ocorre uma concentração de tensões nas áreas próximas ao ponto de aplicação da carga. A quantidade de substrato afetado por esta concentração local é maior dependendo do módulo de elasticidade do material interposto ou do agente de união. Como a espessura da camada adesiva é a mesma para ambos os testes, nos ensaios de microcisalhamento existe uma quantidade proporcionalmente maior deste material com baixo módulo de elasticidade na interface, e assim, esta condição tende a diminuir a concentração de tensões na área mais crítica do modelo [14, 21]. Embora o estudo seja pontual ou represente forças adesivas quase que imediatas, serve como ponto de partida para uma posterior análise de degradação dessa junta adesiva. A biodegradação da interface adesivo/resina gera um espaço entre o substrato dentário e o compósito, ocasionando assim, diminuição das forças adesivas, falhas da restauração e microinfiltrações [25]. Essas microinfiltrações permitem a passagem de agentes patogênicos como as bactérias cariogênicas que geram as lesões de cárie secundárias ou reincidentes. Sendo assim, por estes motivos e pelos adesivos atuais proporcionarem forças adesivas imediatas bastante elevadas, mas que enfraquecem ao longo do tempo, tal tecnologia continua em desenvolvimento constante [26].

\section{CONCLUSÃO}

Tendo em vista as análises efetuadas no presente artigo e os aspectos estudados, pode-se concluir que:

Os valores de $\tau_{\text {nom }}$ obtidos nos ensaios de cisalhamento e microcisalhamento não representam as tensões máximas encontradas durante a simulação por elementos finitos. Porém, por meio das tensões principais pôde-se inferir que as fraturas partem do sentido da aplicação da carga e ao se calcular o $\tau_{\text {máx }}$ para os ensaios de microcisalhamento são obtidos valores máximos muito próximos aos valores nominais, tanto para o modelo bi ou tridimensional.

Não houve variação significativa entre as respostas do MEF para os modelos bi e tridimensionais em relação à interface estudada. Sendo assim, os estudos podem ser simplificados a uma análise bidimensional.

A análise para cisalhamento evidencia que houve falha por tração na dentina, uma vez que as coordenadas estão fora da zona segura, ou seja, a dentina fratura por tração. Já para microcisalhamento, a coordenadas se encontram na zona segura do gráfico, ou seja, a dentina não fratura. Embora dentro da região de tração, esta característica denota que provavelmente estes pontos estão relacionados à resistência adesiva e, portanto, seria mais um fator de validação do teste.

\section{AGRADECIMENTOS}

O presente trabalho foi realizado com apoio da Coordenação de Aperfeiçoamento de Pessoal de Nível Superior - Brasil (CAPES) - Código de Financiamento 001.

\section{BIBLIOGRAFIA}

[1] ORÉFICE, R.L., DISCACCIATI, J.A.C., NEVES, A.D., et al. "In situ evaluation of the polymerization kinetics and corresponding evolution of the mechanical properties of dental composites". Polymer Testing, v. 22, pp. 77-81, 2003.

[2] BAYNE, S.C., HEYMANN, H.O., SWIFT, E.J. "Update on dental composite restoration". The Journal of the American Dental Association, v. 125, n. 6, pp. 687-701, 1994.

[3] TURP, V., SEN, D., TUNCELLI, B., et al. "Adhesion of 10-MDP containing resin cements to dentin with and without the etch-and-rinse technique". The Journal of Advanced Prosthodontics, v. 5, n.1, pp. 226233, 2013.

[4] BAHGAT, H.A., ALSHWAIMI, E., EL-EMBABY, A.E. "Evaluation of the bonding ability of a nanostructured adhesive system”. International Journal of Dental Science and Research, v.2, n.2, pp. 34-40, 2015.

[5] VAN NOORT, R., NOROOZI, S., HOWARD, I.C. et al. "A critique of bond strenght measurements." Journal of Dentistry, v. 17, pp. 61-67, 1989.

[6] GORACCI, C., CURY, A.H., CANTORO, A. et al. "Microtensile bond strenght and interfacial properties of self-etching and self-adhesive resin cements used to lute composite onlays under different seating forces". The Journal of Adhesive Dentistry, v. 8, n. 5, pp. 327-335, 2006. 
[7] SUDSANGIAM S., VAN NOORT, R. “Do dentin bond strength tests serve a useful purpose?” The Journal of Adhesive Dentistry, v.1, n.1, pp. 57-67, 1999.

[8] TANTBIROJN, D., CHENG, Y.S., VERSLUIS, A., et al. "Nominal shear or fracture mechanisms in the assessment of composite-dentin adhesion?” Journal of Dental Research, v. 79, n. 1, pp. 41-48, 2000.

[9] ANUSAVICE, K.J. Screening tests for metal-ceramic systems. In: McLEAN, J.W. pp. 371-414. Proceedings of the First International Symposium on Ceramics. Chicago, Ed. Dental Ceramics, 1983.

[10] DE HOFF, P.H., ANUSAVICE K.J., WANG, Z. "Three-dimensional finite element analysis of the shear bond test". Dental Materials, v. 11, n 2, pp. 126-131, 1995.

[11] SCHERRER, S.S., CESAR, P.F., SWAIN, M.V. "Direct comparison of the bond strength results of the different test methods: A critical literature review". Dental Materials, v. 26, pp. 78-93, 2010.

[12] GRZEBIELUCH, W., BEDZINSKI, R., CZAPLINSKI, T., et al. The mechanical properties of human dentin for 3-D finite element modeling: Numerical and analytical evaluation. Advances in Clinical and Experimental Medicine, v.26, n.4, pp. 645-653, 2017.

[13] PLÁCIDO, E., MEIRA, J.B.C.; LIMA, R.G. et al. "Shear versus micro-shear bond strength test: A finite element stress analysis". Dental Materials, v. 23, pp. 1086-1092, 2007.

[14] VAN NOORT, R., CARDEW, G.E., HOWARD, I.C. "A study of the interfacial shear and tensile stresses in a restored molar tooth”. Journal of Dentistry, v. 16, pp. 286-293, 1988.

[15] AUSIELLO, P., APICELLA, A., DAVIDSON C.L. "Effect of adhesive layer properties on stress distribution in composite restorations - a 3D finite element analysis". Dental Materials, v. 18, pp. 295-303, 2002.

[16] YU, M. "Advances in strength theories for materials under complex stress state in the 20th Century". Applied Mechanics Reviews, v. 55, n. 3; pp.169-218, 2002.

[17] VAN NOORT, R., CARDEW, G.E., HOWARD, I.C. et al. "The effect of local interfacial geometry on the measurement of the tensile bond strenght to dentin". Journal of Dental Research, v. 70, n. 3, pp. 889-893, 1991.

[18] XAVIER, T.A., MEIRA, J.B.C., RODRIGUES, F.P. et al. "Finite element analysis of shear versus torsion adhesive strength tests for dental resin composites". Journal of Adhesion Science and Technology, v. 23, pp. 1575-1589, 2009.

[19] JIN, X., HOAMEI, E., MATINLINNA, J.P., et al. “A new concept and finite-element study on dental bond strength tests". Dental Materials, v. 32, pp. 238-250, 2016.

[20] MISRA, A., SPENCER P., MARANGOS, O., et al. "Micromechanical analysis of dentin/adhesive interface by the finite element method". Journal of Biomedical Materials Research Part B: Applied Biomaterials, v. 70, n. 1, pp. 56-65, 2004.

[21] XAVIER, T.A., BALLESTER, R.Y. "A comparison between the capacity of 2D and 3D finite element models in analyzing the stress distribution in shear and microshear bond strength tests". Journal of Research in Dentistry, v. 1, n. 1, pp. 41-54, 2013.

[22] JUVINAL, R.C., MARSHEK, K.M. "Fundamentals of machine component design". 2a ed. New York: Wiley Sons, 1991, 217 p.

[23] SAKAGUCHI, R.L., POWERS, J.M. Craig, materiais dentários restauradores, 13 ed., Rio de Janeiro, Elsevier, 2012.

[24] ESCRIBANO, N.I., DEL-NERO, M.O., MACORRA, J.C. "Inverse relationship between tensile bond strength and dimensions of bonded área". Journal of Biomedical Materials Research, v. 66, pp. 419-424, 2003.

[25] LIU, Y., TJÄDERHANE, L., BRESCHI, L., et al. "Limitations in Bonding to Dentin and Experimental Strategies to Prevent Bond Degradation”. Journal of Dental Research, v. 90, n. 8, pp. 953-968, 2011.

[26] PERDIGÃO, J., REIS, A., LOGUERCIO, A.D. "Dentin Adhesion and MMPs: A Comprehensive Review”, Journal of Esthetic and Restorative Dentistry, v. 25 n. 4, 2013.

ORCID

Eric Costa Carvalho Jayme Pereira de Gouvêa https://orcid.org/0000-0001-9169-6303

https://orcid.org/0000-0003-0045-4873 
Ágatha Borges Teixeira

Cláudio Luís de Melo-Silva

Tereza Cristina Favieri de Melo-Silva https://orcid.org/0000-0003-0988-0844

https://orcid.org/0000-0001-5093-2189

https://orcid.org/0000-0001-5309-7190 